\title{
Frequency downshift in a viscous fluid
}

\author{
John D. Carter ${ }^{\mathrm{a}, *}$, Alex Govan ${ }^{\mathrm{a}}$ \\ ${ }^{a}$ Mathematics Department, Seattle University, Seattle, WA 98122
}

\begin{abstract}
In this paper, we derive a viscous generalization of the Dysthe [1] system from the weakly viscous generalization of the Euler equations introduced by Dias, Dyachenko, \& Zakharov [2]. This "viscous Dysthe" system models the evolution of a weakly viscous, nearly monochromatic wave train on deep water. It includes only one free parameter, which can be determined empirically. It contains a term that provides a mechanism for frequency downshifting in the absence of wind and wave breaking. The system does not preserve the spectral mean. Numerical simulations demonstrate that the spectral mean typically decreases and that the spectral peak decreases for certain initial conditions. The linear stability analysis of the plane-wave solutions of the viscous Dysthe system demonstrates that waves with frequencies closer to zero decay more slowly than waves with frequencies further from zero. Comparisons between experimental data and numerical simulations of the nonlinear Schrödinger, dissipative nonlinear Schrödinger, Dysthe, and viscous Dysthe systems establish that the viscous Dysthe system accurately models data from experiments in which frequency downshifting was observed and experiments in which frequency downshift was not observed.
\end{abstract}

\section{Introduction}

In the late 1970s, Lake et al. [3] and Lake \& Yuen [4] conducted physical experiments that investigated the evolution of nonlinear wave trains on deep water. They found that the growth of the Benjamin-Feir instability is followed by a shift in the spectral peak, the frequency corresponding to the Fourier mode with largest magnitude, to a frequency closer to zero. Subsequent experiments, including those in Su et al. [5] and Melville [6], demonstrated that the amplitude of the lower sideband grows and eventually overtakes that of the carrier wave. These later experimental studies focused on waves with larger steepness and involved wave breaking. More recently, Segur et al. [7] conducted similar experiments without wave breaking or wind. They found that frequency downshifting (FD) is not observed (in their tank) if the waves have what they refer to as "small or moderate" amplitudes and that FD is observed if the amplitude of

\footnotetext{
${ }^{*}$ Corresponding author
} 
the carrier wave is "large" or if the sideband perturbations are "large enough." They also found that if FD occurs then (i) the spectral mean (defined below) decreases monotonically as the waves travel down the tank and (ii) FD occurs in the higher harmonics before it occurs in the fundamental. The goal of the current work is to provide a mathematical justification for FD that does not rely on wind or wave breaking.

There are two quantities that are commonly used to quantify frequency downshifting: the spectral peak, $\omega_{p}$, and the spectral mean, $\omega_{m}$. The spectral mean (in Hertz) at a location $x$ in tank is given by

$$
\omega_{m}(x)=\frac{\mathcal{P}(x)}{\mathcal{M}(x)},
$$

where $\mathcal{M}$ (in $\mathrm{cm}^{2}$ ) and $\mathcal{P}$ (in $\mathrm{cm}^{2} / \mathrm{sec}$ ) are defined by

$$
\begin{gathered}
\mathcal{M}(x)=\frac{1}{L} \int_{0}^{L}|B|^{2} d t \\
\mathcal{P}(x)=\frac{i}{2 L} \int_{0}^{L}\left(B B_{t}^{*}-B_{t} B^{*}\right) d t .
\end{gathered}
$$

Here $B$ (in $\mathrm{cm})$ is a measure of the slow evolution of the complex envelope of a nearly monochromatic train of plane waves; $x$ (in $\mathrm{cm}$ ) is the horizontal coordinate in the direction of wave propagation; $t$ (in sec) is the temporal coordinate; $L$ (in sec) is the $t$ period of $B$; and ${ }^{*}$ represents complex conjugate. We note that while some studies use $\mathcal{P}$ by itself as a measure of FD, we focus on $\omega_{p}$ and $\omega_{m}$.

Zakharov [8] derived the cubic nonlinear Schrödinger (NLS) equation from the Euler equations as a model for the evolution of the envelope of a nearly monochromatic wave group. The NLS equation preserves the spectral mean, so it cannot be used to model FD. Dysthe [1] carried out the NLS perturbation analysis one order higher to obtain what is now known as the Dysthe system. Lo \& Mei [9] numerically solved the NLS equation and Dysthe system and established that the Dysthe system more accurately predicts the evolution of mildly sloped, narrow-banded, weakly nonlinear waves over longer time periods than does the NLS equation. They also found that dissipative generalizations of the Dysthe system are required to model waves of moderate steepness over long distances. Finally, their numerical studies showed that the Dysthe system did not lead to a permanent FD even though the Dysthe system does not preserve the spectral mean. Segur et al. [7] established that the dissipative NLS equation accurately models the evolution of waves trains in which no FD occurred and that it cannot model FD because it preserves the spectral mean. The dissipative NLS equation was used as an ad-hoc model without formal justification until Dias, Dyachenko, \& Zakharov [2] derived it from a weakly viscous generalization of the Euler equations. The first step in the current work is to carry out the Dysthe perturbation analysis starting from the Dias, Dyachenko, \& Zakharov weakly viscous generalization of the Euler equations in order to derive a new 
system, which we call the viscous Dysthe system. Readers interested in wind and wave-breaking justifications for FD are referred to Trulsen \& Dysthe [10], Hara \& Mei [11], Kato \& Oikawa [12], Brunetti \& Kasparian [13], Brunetti et al. [14], and Islas \& Schober [15].

The paper is organized as follows: Section 2 contains the derivation of the viscous Dysthe system. Section 3 contains a summary of the properties of this new equation. Section 4 contains comparisons of viscous Dysthe predictions with experimental data from two experiments, ne of which exhibited FD.

\section{Derivation of the viscous Dysthe equation}

Dias, Dyachenko, \& Zakharov [2] introduced the following system for an infinitely-deep, weakly viscous fluid

$$
\begin{gathered}
\phi_{x x}+\phi_{y y}+\phi_{z z}=0, \quad \text { for }-\infty<z<\eta, \\
\phi_{t}+\frac{1}{2}|\nabla \phi|^{2}+g \eta=-2 \bar{\nu} \phi_{z z}, \quad \text { at } z=\eta, \\
\eta_{t}+\eta_{x} \phi_{x}+\eta_{y} \phi_{y}=\phi_{z}+2 \bar{\nu} \Delta \eta, \quad \text { at } z=\eta, \\
|\nabla \phi| \rightarrow 0, \quad \text { as } z \rightarrow-\infty .
\end{gathered}
$$

Here $\phi=\phi(x, y, z, t)$ represents the velocity potential of the fluid; $\eta=\eta(x, y, t)$ represents the free-surface displacement; $t$ is the temporal coordinate; $x$ and $y$ are horizontal coordinates; $z$ is the vertical coordinate; $g$ represents the acceleration due to gravity; and $\bar{\nu}>0$ represents the kinematic viscosity of the fluid. This model assumes that gravity and viscosity are the only forces acting on the fluid. The Euler equations are obtained from (3) by setting $\bar{\nu}=0$.

As frequency downshifting is most commonly observed in time series recorded at various locations in the direction of wave propagation, assume

$$
\begin{gathered}
\eta(x, y, t)=\epsilon^{3} \bar{\eta}+\epsilon B \mathrm{e}^{i \omega_{0} t-i k_{0} x}+\epsilon^{2} B_{2} \mathrm{e}^{2\left(i \omega_{0} t-i k_{0} x\right)}+\cdots+c . c . \\
\phi(x, y, z, t)=\epsilon^{2} \bar{\phi}+\epsilon A_{1} \mathrm{e}^{k_{0} z+i \omega_{0} t-i k_{0} x}+\epsilon^{2} A_{2} \mathrm{e}^{2\left(k_{0} z+i \omega_{0} t-i k_{0} x\right)}+\cdots+c . c .
\end{gathered}
$$

where $\omega_{0}>0$ and $k_{0}>0$ represent the frequency and wave number of the carrier wave respectively; $\epsilon=2 a_{0} k_{0} \ll 1$ is a dimensionless parameter known as the wave steepness; $a_{0}$ represents a typical wave amplitude; and c.c. stands for complex conjugate. Assuming $k_{0}>0$ determines the $z$ dependence in $\phi$. The choice $\omega_{0}>0$ implies that the wave train travels in the positive $x$ direction as $t$ increases. The $A$ 's and $\bar{\phi}$ depend on the slow variables $X=\epsilon x, Y=\epsilon Y$, $Z=\epsilon z$, and $T=\epsilon t$, while the $B$ 's and $\bar{\eta}$ depend on $X, Y$ and $T$. Next, assume

$$
\begin{gathered}
A_{j}=A_{j 0}+\epsilon A_{j 1}+\epsilon^{2} A_{j 2}+\epsilon^{3} A_{j 3}+\ldots, \\
B_{j}=B_{j 0}+\epsilon B_{j 1}+\epsilon^{2} B_{j 2}+\epsilon^{3} B_{j 3}+\ldots, \quad \text { for } j=1,2,3, \ldots, \\
\bar{\eta}=\bar{\eta}_{0}+\epsilon \bar{\eta}_{1}+\epsilon^{2} \bar{\eta}_{2}+\ldots,
\end{gathered}
$$




$$
\bar{\phi}=\bar{\phi}_{0}+\epsilon \bar{\phi}_{1}+\epsilon^{2} \bar{\phi}_{2}+\ldots
$$

Following the work of Dias, Dyachenko, \& Zakharov, assume that viscous effects are small by setting $\bar{\nu}=\epsilon^{2} \nu$. Substituting (4) and (5) into (3) and completing the fourth-order perturbation analysis gives the deep-water dispersion relationship,

$$
g k_{0}=\omega_{0}^{2},
$$

the system that defines $B$ and $\bar{\phi}_{0}$,

$$
\begin{gathered}
2 i \omega_{0}\left(B_{T}+\frac{g}{2 \omega_{0}} B_{X}\right)+\epsilon\left(\frac{g}{4 k_{0}} B_{X X}-\frac{g}{2 k_{0}} B_{Y Y}+4 g k_{0}^{3}|B|^{2} B+4 i k_{0}^{2} \omega_{0} \nu B\right) \\
+\epsilon^{2}\left(-\frac{i g}{8 k_{0}^{2}} B_{X X X}+\frac{3 i g}{4 k_{0}^{2}} B_{X Y Y}+2 i g k_{0}^{2} B^{2} B_{X}^{*}\right. \\
\left.+12 i g k_{0}^{2}|B|^{2} B_{x}+2 k_{0} \omega_{0} B \bar{\phi}_{0 X}-8 k_{0} \omega_{0} \nu B_{X}\right)=0, \text { at } Z=0 \\
\bar{\phi}_{0 Z}=2 \omega_{0}\left(|B|^{2}\right)_{X}, \quad \text { at } Z=0, \\
\bar{\phi}_{0 X X}+\bar{\phi}_{0 Z Z}=0, \quad \text { for }-\infty<Z<0 \\
\bar{\phi}_{0 Z} \rightarrow 0, \quad \text { as } Z \rightarrow-\infty
\end{gathered}
$$

and

$$
\begin{gathered}
B_{2}=k_{0} B^{2}+\mathcal{O}(\epsilon), \\
B_{3}=\frac{3}{2} k_{0}^{2} B^{3}+\mathcal{O}(\epsilon) .
\end{gathered}
$$

Equations (8a) and (8b) define the leading-order contributions to the amplitudes of the second and third harmonics of the carrier wave respectively.

Interchanging the forms of the assumptions in equations (5a) and (5b) and following a similar procedure while focusing on the leading-order term of the velocity potential instead of the leading-order term of the surface displacement gives

$$
\begin{gathered}
2 i \omega_{0}\left(A_{T}+\frac{g}{2 \omega_{0}} A_{X}\right)+\epsilon\left(\frac{g}{4 k_{0}} A_{X X}-\frac{g}{2 k_{0}} A_{Y Y}+4 k_{0}^{4}|A|^{2} A+4 i k_{0}^{2} \omega_{0} \nu A\right) \\
+\epsilon^{2}\left(-\frac{i g}{8 k_{0}^{2}} A_{X X X}+\frac{3 i g}{4 k_{0}^{2}} A_{X Y Y}-2 i k_{0}^{3} A^{2} A_{X}^{*}\right. \\
\left.+12 i k_{0}^{3}|A|^{2} A_{x}+2 k_{0} \omega_{0} A \bar{\phi}_{0 X}-8 k_{0} \omega_{0} \nu A_{X}\right)=0, \text { at } Z=0, \\
\bar{\phi}_{0 Z}=\frac{2 k_{0}^{2}}{\omega_{0}}\left(|A|^{2}\right)_{X}, \quad \text { at } Z=0, \\
\bar{\phi}_{0 X X}+\bar{\phi}_{0 Z Z}=0, \quad \text { for }-\infty<Z<0, \\
\bar{\phi}_{0 Z} \rightarrow 0, \quad \text { as } Z \rightarrow-\infty .
\end{gathered}
$$

In essence, this system defines $A_{1}$ in equation (5a). 
The systems in (7) and (9) are related by

$$
\begin{gathered}
B=-\frac{i k_{0}}{\omega_{0}} A+\epsilon \frac{1}{2 \omega_{0}} A_{X}+\epsilon^{2}\left(-\frac{i}{8 k_{0} \omega_{0}} A_{X X}+\frac{i}{4 k_{0} \omega_{0}} A_{Y Y}-\frac{i k_{0}^{4}}{2 g \omega_{0}}|A|^{2} A\right)+\mathcal{O}\left(\epsilon^{3}\right), \\
A=\frac{i \omega_{0}}{k_{0}} B+\epsilon \frac{\omega_{0}}{2 k_{0}^{2}} B_{X}+\epsilon^{2}\left(-\frac{3 i \omega_{0}}{8 k_{0}^{3}} B_{X X}+\frac{i \omega_{0}}{4 k_{0}^{3}} B_{Y Y}-\frac{i k_{0} \omega_{0}}{2}|B|^{2} B\right)+\mathcal{O}\left(\epsilon^{3}\right) .
\end{gathered}
$$

In order to nondimensionalize and enter into a coordinate frame moving with the linear group velocity, $\frac{\omega_{0}}{2 k_{0}}$, apply the following change of variables. For simplicity, assume that there is no $Y$ dependence. Let

$$
\begin{gathered}
k_{0} B(X, Y, T)=\tilde{B}(\xi, \chi), \\
\frac{k_{0}^{2}}{\omega_{0}} A(X, Y, Z, T)=\tilde{A}(\xi, \chi, \zeta), \\
\frac{k_{0}^{2}}{4 \omega_{0}} \bar{\phi}_{0}(X, Y, Z, T)=\tilde{\Phi}(\xi, \chi, \zeta), \\
\frac{4 k_{0}^{2}}{\omega_{0}} \nu=\delta, \\
\chi=\epsilon k_{0} X, \\
\xi=\omega_{0} T-2 k_{0} X, \\
\zeta=k_{0} Z .
\end{gathered}
$$

This change of variables leads to (where tildes have been dropped for convenience)

$$
\begin{array}{r}
i B_{\chi}+B_{\xi \xi}+4|B|^{2} B+i \delta B+\epsilon\left(-8 i B^{2} B_{\xi}^{*}-32 i|B|^{2} B_{\xi}\right. \\
\left.-16 B \Phi_{\xi}+5 \delta B_{\xi}\right)=0, \text { at } \zeta=0, \\
\Phi_{\zeta}=-\left(|B|^{2}\right)_{\xi} \quad \text { at } \zeta=0, \\
4 \Phi_{\xi \xi}+\Phi_{\zeta \zeta}=0, \quad \text { for }-\infty<\zeta<0, \\
\Phi_{\zeta} \rightarrow 0, \quad \text { as } \zeta \rightarrow-\infty,
\end{array}
$$

and

$$
\begin{gathered}
i A_{\chi}+A_{\xi \xi}+4|A|^{2} A+i \delta A+\epsilon\left(-32 i|A|^{2} A_{\xi}-16 A \Phi_{\xi}\right. \\
\left.+5 \delta A_{\xi}\right)=0, \text { at } \zeta=0, \\
\Phi_{\zeta}=-\left(|A|^{2}\right)_{\xi}, \quad \begin{array}{c}
\text { at } \zeta=0, \\
4 \Phi_{\xi \xi}+\Phi_{\zeta \zeta}=0,
\end{array} \\
\Phi_{\zeta} \rightarrow 0, \quad \text { for }-\infty<\zeta<0,
\end{gathered}
$$


We call the systems given in equations (12) and (13) the viscous Dysthe system for the surface displacement (the $\mathrm{vB}$ system) and the viscous Dysthe system for the velocity potential (the vA system) respectively. These equations describe the evolution of a narrow-banded, weakly nonlinear, weakly viscous fluid. The NLS equation is obtained from equation (12a) or (13a) by setting $\delta=\epsilon=0$. The Dysthe systems for the surface displacement (the DB system) and velocity potential (the DA system) are obtained by setting $\delta=0$ in equations (12) and (13) respectively.

It is important to note that there is only one free parameter in the viscous Dysthe systems. This parameter, $\delta$, is empirically determined by fitting the decay of $\mathcal{M}$. This makes the viscous Dysthe systems qualitatively different than other FD models that have two (or more) free parameters that can be used to fit $\mathcal{M}, \mathcal{P}$, or other quantities.

\section{Properties of the viscous Dysthe system}

\subsection{Mass and linear momentum}

Using the variables introduced in equations (4) and (11), the quantities $\mathcal{M}$ and $\mathcal{P}$ can be rewritten as

$$
\begin{gathered}
\mathcal{M}(\chi)=\frac{\epsilon^{2}}{k_{0}^{2}} \frac{1}{\epsilon \omega_{0} L} \int_{0}^{\epsilon \omega_{0} L}|B|^{2} d \xi \\
\mathcal{P}(\chi)=\frac{\epsilon^{3} \omega_{0}}{k_{0}^{2}} \frac{i}{2 \epsilon \omega_{0} L} \int_{0}^{\epsilon \omega_{0} L}\left(B B_{\xi}^{*}-B_{\xi} B^{*}\right) d \xi .
\end{gathered}
$$

Trulsen \& Dysthe [16] showed that the DA system preserves both $\mathcal{M}$ and $\mathcal{P}$ in $\chi$, while the DB system preserves $\mathcal{M}$, but not $\mathcal{P}$. Moreover, they established that the sign of $\mathcal{P}_{\chi}$ in DB system is not definite and therefore the Dysthe system can predict frequency upshifting. Lo \& Mei [9] conducted numerical studies of the DB system and did not observe a permanent downshift in any of their simulations. These results suggest that the $B^{2} B_{\xi}^{*}$ term (the only term that differentiates the DB and DA systems) is not the mechanism for FD.

The DA system is Hamiltonian while the DB system system is likely not Hamiltonian (see, for example, Carter [17] and Segur, Henderson, \& Hammack [18]). Gramstad \& Trulsen [19] derived a Hamiltonian generalization of the VB system. Although we do not study this equation here, we expect that the results shown below will generalize to it because the viscosity term, $5 \epsilon \delta B_{\xi}$, provides the mechanism for FD.

The $\chi$ dependencies of $\mathcal{M}$ and $\mathcal{P}$ in the vB system are given by

$$
\begin{gathered}
\mathcal{M}_{\chi}=-2 \delta \mathcal{M}-10 \frac{\delta}{\omega_{0}} \mathcal{P}, \\
\mathcal{P}_{\chi}=-2 \delta \mathcal{P}-10 \frac{\delta}{\omega_{0}} \mathcal{Q}-\frac{16}{\omega_{0}} \mathcal{R},
\end{gathered}
$$


where

$$
\begin{gathered}
\mathcal{Q}=\frac{\epsilon^{4} \omega_{0}^{2}}{k_{0}^{2}} \frac{1}{\epsilon \omega_{0} L} \int_{0}^{\epsilon \omega_{0} L}\left|B_{\xi}\right|^{2} d \xi \\
\mathcal{R}=\frac{\epsilon^{4} \omega_{0}^{2}}{k_{0}^{2}} \frac{1}{\epsilon \omega_{0} L} \operatorname{Im}\left(\int_{0}^{\epsilon \omega_{0} L}|B|^{2} B^{*} B_{\xi \xi} d \xi\right) .
\end{gathered}
$$

Further

$$
\left(\omega_{m}\right)_{\chi}=\left(\frac{\mathcal{P}}{\mathcal{M}}\right)_{\chi}=-\frac{10 \delta}{\omega_{0} \mathcal{M}^{2}}\left(\mathcal{M} \mathcal{Q}-\mathcal{P}^{2}\right)-\frac{16}{\omega_{0}} \frac{\mathcal{R}}{\mathcal{M}} .
$$

Equation (15a) shows that $\mathcal{M}$ decreases more rapidly when $\mathcal{P}>0$ and more slowly when $\mathcal{P}<0$. This suggests a preference for waves with negative wave number in $\xi$. The first term on the right-hand side of (15b) establishes that to leading-order the absolute value of $\mathcal{P}$ decreases in $\chi$. The second term causes $\mathcal{P}$ to decrease regardless of the sign of $\mathcal{P}$. Unfortunately, no general statements can be made about the $\mathcal{R}$ term, which arises in the viscosity-free DB system, because it does not have a definite sign [16].

The Cauchy-Schwarz inequality establishes that $\left(\mathcal{M Q}-\mathcal{P}^{2}\right) \geq 0$. This establishes that viscosity $(\delta>0)$ causes the spectral mean to decrease. The sign of the $\mathcal{R} / \mathcal{M}$ term can vary, so FD in the spectral mean sense is not necessarily guaranteed. Finally, equation (17) establishes that FD is a higher-order in $\epsilon$ effect. This corroborates the Segur et al. result that the dissipative NLS equation preserves the spectral mean.

\subsection{Plane-Wave Solutions}

Consider solutions of the vB system with the following form

$$
B(\xi, \chi)=B_{0} \exp \left(i l \xi+w_{r}(\chi)+i w_{i}(\chi)+i \rho\right),
$$

where $B_{0}, l$, and $\rho$ are real constants and $w_{r}$ and $w_{i}$ are real-valued functions. This gives

$$
\begin{gathered}
\Phi(\xi, \chi, \zeta)=0, \\
w_{r}^{\prime}(\chi)+\delta(1+5 \epsilon l)=0, \\
w_{i}^{\prime}(\chi)+l^{2}-4 B_{0}^{2}(1+6 \epsilon l) \mathrm{e}^{2 w_{r}(\chi)}=0 .
\end{gathered}
$$

The parameter $\rho$ does not play a role in the ODEs that define $\omega_{r}$ and $\omega_{i}$ because of the gauge invariance of the vB system. This allows all complex $B_{0}$ to be considered by assuming, without loss of generality, that $B_{0}$ is a positive constant. The solution of this system is

$$
\begin{gathered}
w_{r}(\chi)=-\delta(1+5 \epsilon l) \chi \\
w_{i}(\chi)=-l^{2} \chi+\frac{2 B_{0}^{2}(1+6 \epsilon l)}{\delta(1+5 \epsilon l)}\left(1-\mathrm{e}^{-2 \delta(1+5 \epsilon l) \chi}\right) .
\end{gathered}
$$

The constants of integration were chosen so that these (viscous) solutions limit to the solutions of the (nonviscous) DB system in the $\delta \rightarrow 0$ limit. Note that 
(20a) establishes that if $\delta>0$, then to leading order all plane-wave solutions of the vB system decay to zero as $\chi \rightarrow \infty$. Just as in the NLS equation and the Dysthe systems, choosing $l \neq 0$ corresponds to shifting the frequency of the carrier wave. The function $\omega_{r}$ depends on $l$ in such a way that it takes into account the correct (up to the order of the equation) rate of dissipation for the shifted carrier wave. Therefore, we assume $l=0$ for the remainder of the paper.

\subsection{Linear stability analysis}

In order to study the stability of solutions of the form given in equation (18), we consider perturbed solutions of the form

$$
\begin{gathered}
B_{\text {pert }}(\xi, \chi)=\left(B_{0}+\mu u(\xi, \chi)+i \mu v(\xi, \chi)\right) \exp \left(w_{r}(\chi)+i w_{i}(\chi)\right), \\
\Phi_{\text {pert }}(\xi, \chi, \zeta)=0+\mu p(\xi, \chi, \zeta),
\end{gathered}
$$

where $\mu$ is a small real parameter; $u, v$, and $p$ are real-valued functions; and $w_{r}$ and $w_{i}$ are given in equation (20). Note that the decay due to $w_{r}$ has been factored out in equation (21a). This allows the evolution of the surface displacement perturbation, $\mu(u+i v)$, to be directly compared to the constant $B_{0}$. Therefore, if $u$ and/or $v$ grow without bound in $\chi$, we say that the solutions is linearly unstable (even though the magnitude of the entire perturbed solution may decay to zero).

Substituting (21) into (12), linearizing in $\mu$, and separating into real and imaginary parts leads to a system of coupled partial differential equations (PDEs) that have constant coefficients in $\xi$ and $\zeta$. Without loss of generality, assume

$$
\begin{gathered}
u(\xi, \chi)=U(\chi) \mathrm{e}^{i q \xi}+U^{*}(\chi) \mathrm{e}^{-i q \xi}, \\
v(\xi, \chi)=V(\chi) \mathrm{e}^{i q \xi}+V^{*}(\chi) \mathrm{e}^{-i q \xi}, \\
p(\xi, \chi, \zeta)=-\operatorname{sign}(q) B_{0}\left(i U(\chi) \mathrm{e}^{i q \xi}-i U^{*}(\chi) \mathrm{e}^{-i q \xi}\right) \mathrm{e}^{2|q| \zeta} \mathrm{e}^{-2 \delta \chi},
\end{gathered}
$$

where $q$ is a real constant, and $U$ and $V$ are complex-valued functions. The form for $p$ given in equation (22c) is used because it satisfies Laplace's equation and the bottom boundary condition. Substituting (22) into the coupled PDEs gives

$$
\left(\begin{array}{l}
U \\
V
\end{array}\right)^{\prime}=\left(\begin{array}{l}
\mathbf{A}_{1}+\mathrm{e}^{2 w_{r}(\chi)} \mathbf{A}_{\mathbf{2}}
\end{array}\right)\left(\begin{array}{l}
U \\
V
\end{array}\right),
$$

where prime means derivative with respect to $\chi$ and $\mathbf{A}_{\mathbf{1}}$ and $\mathbf{A}_{\mathbf{2}}$ are the constant matrices defined by

$$
\begin{aligned}
& \mathbf{A}_{\mathbf{1}}=\left(\begin{array}{cc}
0 & q^{2}-5 i \epsilon \delta q \\
-q^{2}+5 i \epsilon \delta q & 0
\end{array}\right), \\
& \mathbf{A}_{\mathbf{2}}=\left(\begin{array}{cc}
40 i \epsilon q B_{0}^{2} & 0 \\
8(1-2 \epsilon|q|) B_{0}^{2} & 24 i \epsilon q B_{0}^{2}
\end{array}\right) .
\end{aligned}
$$

The classical instability result for the NLS equation, i.e. any $q$ satisfying $q^{2}<$ $8 B_{0}^{2}$ gives instability, is obtained by solving equation (23) with $\epsilon=\delta=0$. To 
our knowledge, an exact solution of (23) when $\epsilon$ and $\delta$ are nonzero is not known. The matrix $\mathbf{A}_{1}$ determines the large- $\chi$ behavior of the solution because the $\chi$ dependent term is integrable on $\chi \in[0, \infty)$ (see, for example, Coddington \& Levinson [20]). The eigenvalues of $\mathbf{A}_{\mathbf{1}}$ are

$$
\lambda_{A_{1}}= \pm i q^{2} \pm 5 \epsilon \delta q .
$$

This establishes that the long-term solution of (23) is given by

$$
\left(\begin{array}{l}
U \\
V
\end{array}\right)=\left(\begin{array}{cc}
-i c_{1} & i c_{2} \\
c_{1} & c_{2}
\end{array}\right)\left(\begin{array}{c}
\exp \left(\left(i q^{2}+5 \epsilon \delta q\right) \chi\right) \\
\exp \left(\left(-i q^{2}-5 \epsilon \delta q\right) \chi\right)
\end{array}\right),
$$

where $c_{1}$ and $c_{2}$ are the constants of integration. Substituting this back into equation (21a) gives

$$
\begin{aligned}
B_{\text {pert }}=\left(B_{0}+2 i \mu c_{1} \exp \right. & \left(-i q \xi-i q^{2} \chi+5 \epsilon \delta q \chi\right) \\
+ & \left.2 i \mu c_{2} \exp \left(i q \xi-i q^{2} \chi-5 \epsilon \delta q \chi\right)\right) \mathrm{e}^{w_{r}(\chi)+i w_{i}(\chi)} .
\end{aligned}
$$

The leading-order surface displacement in physical coordinates corresponding to this perturbed plane-wave solution of the vB system is

$$
\begin{aligned}
\eta(x, t)=d_{0} \exp \left(i \omega_{0} t+i f_{0}(x)-4 \bar{\nu} \frac{k_{0}^{3}}{\omega_{0}} x\right) \\
+d_{1} \exp \left(i \omega_{0}(1-\epsilon q) t+i f_{1}(x)-4 \bar{\nu} \frac{k_{0}^{3}}{\omega_{0}}(1-5 \epsilon q) x\right) \\
\quad+d_{2} \exp \left(i \omega_{0}(1+\epsilon q) t+i f_{2}(x)-4 \bar{\nu} \frac{k_{0}^{3}}{\omega_{0}}(1+5 \epsilon q) x\right)+\text { c.c. }
\end{aligned}
$$

where the $d_{j}$ are complex constants and the $f_{j}$ are real-valued functions.

\section{Observations:}

- If $q \neq 0$ then $\mathbf{A}_{1}$ has an eigenvalue with positive real part in the viscous $(\delta>0)$ case. Therefore all plane-wave solutions of the $\mathrm{vB}$ system are linearly unstable. The growth rate of the instability is $5 \epsilon \delta|q|$. This instability is not a Benjamin-Feir-like instability because all spatially dependent perturbations lead to exponential growth. This instability is similar to the "enhanced Benjamin-Feir" instabilities that arise in other dissipative generalizations of the NLS equation (see, for example, Bridges \& Dias [21] or Carter \& Contreras [22]).

- If $q$ is positive, then the $c_{1}$ term in (27) grows exponentially in $\chi$ while the $c_{2}$ term decays exponentially in $\chi$. If $q$ is negative, then the $c_{2}$ term grows while the $c_{1}$ term decays. The fact that perturbations with negative wave numbers grow while perturbations with positive wave numbers decay suggests FD. 
- In equation (27) the decay due to $\omega_{r}$ has been factored out. This decay is an $\mathcal{O}(1)$ effect, while the growth of the instability is an $\mathcal{O}(\epsilon)$ effect. Although this means decay typically dominates the growth, it also means that some modes decay more slowly than others.

- Equation (28) shows that the amplitude of the carrier wave (the wave with frequency $\omega_{0}>0$ ) decays exponentially in $x$.

- If $q>0$, then the amplitude of the wave with frequency $\omega_{0}(1-\epsilon q)$ (the lower sideband) decays more slowly in $x$ than the amplitude of the carrier wave. The mode with frequency $\omega_{0}(1+\epsilon q)$ (the upper sideband) decays more rapidly in $x$ than the carrier wave. This suggests FD.

- If $q<0$, then the mode with frequency $\omega_{0}(1-\epsilon q)$ (the upper sideband) decays more rapidly in $x$ than the carrier wave. The mode with frequency $\omega_{0}(1+\epsilon q)$ (the lower sideband) decays more slowly in $x$ than the carrier wave. This suggests FD.

- Repeating these calculations for the $B^{*}$ term in (4) show that the wave with frequency $-\omega_{0}(1+\epsilon|q|)$ decays more rapidly than the carrier wave (the wave with frequency $-\omega_{0}<0$ ) which decays more rapidly than the wave with frequency $-\omega(1-\epsilon|q|)$. These results further establish that waves with frequencies closer to zero decay more slowly than waves with frequencies further from zero.

- The growth rate $5 \epsilon \delta|q|$ and equation (8a) establish that the instability in the second harmonic (the $B_{2}$ term in (4a)) grows twice as fast as the instability in the fundamental (the $B$ term in (4a)). This suggests that FD will be observed in the higher harmonics before it is observed in the fundamental (just as was observed by Segur et al.)

\section{Comparisons with experiments}

In this section we test the validity of the $\mathrm{vB}$ system by comparing its predictions with data from two physical experiments of one-dimensional, nearly monochromatic wave trains on deep water and predictions from the NLS, dissipative NLS, and Dysthe equations. The waves were created by a plunger-type wave maker that oscillates vertically at one end of a 43 -foot long and 10-inch wide wave channel. Waves traveled in only one direction, toward increasing $x$. Time series were collected by eleven or twelve (depending on the experiment) wave gauges located $128+50(m-1) \mathrm{cm}$ from the wave maker were $m=1, \ldots, 12$. The tank was long enough that reflections off the far wall did not play a role. The data come from the experiments originally presented in Segur et al. [7]. Complete experimental details are found in Section 6 of that paper.

The first experiment examines the evolution of a nearly monochromatic wave train formed by a carrier wave perturbed by sidebands of "moderate" amplitude. The second experiment examines the evolution of a nearly monochromatic wave 
train formed by a very similar carrier wave perturbed by sidebands of "large" amplitude. FD was observed in the second experiment, but not in the first. For each experiment, the wave gauges recorded time series lasting 23.4 seconds. The experimental initial conditions were comprised of a carrier wave with a frequency of $3.33 \mathrm{~Hz}\left(\omega_{0}=20.9 \mathrm{rad} / \mathrm{sec}, k_{0}=0.447 \mathrm{~cm}^{-1}\right)$ and one upper and one lower seeded sideband each separated from the carrier wave by $0.171 \mathrm{~Hz}$ $(\Delta \omega=1.07 \mathrm{rad} / \mathrm{sec})$.

All model PDEs were solved numerically by assuming periodic boundary conditions in $\xi$ and using split-step pseudospectral methods that allow the linear parts of the PDEs to be solved exactly in Fourier space. The initial conditions for the PDEs were

$$
B(\xi, \chi=0)=\frac{k_{0}}{\epsilon} \sum_{n=-3}^{3} a_{n} \mathrm{e}^{i n \xi /(78 \epsilon)} .
$$

Here $a_{0}$ represents the experimentally-measured complex amplitude of the carrier wave, $a_{-1}$ represents the amplitude of the first lower sideband, etc., measured at the first wave gauge. This form was selected for the initial conditions because it contains all of the waves with significant amplitudes at the first gauge.

\subsection{Moderate-amplitude experiment}

Table 1 contains the values of the physical parameters for this experiment. Figure 1 contains plots of the Fourier amplitudes versus $x$, the distance from the first wave gauge in centimeters. Unlike the plots and data in Segur et al., the data here are not scaled. Figure 2 shows how $\mathcal{M}, \mathcal{P}, \omega_{p}$, and $\omega_{m}$ evolved as the wave train progressed down the tank. Although the amplitudes of the sidebands increase, the amplitude of the carrier wave is dominant at all measurement sites, so the experimental spectral peak was constant. Additionally, the experimental spectral mean was constant to within experimental error. Therefore, neither FD in the spectral peak nor in spectral mean sense occurred in this experiment.

The leading-order version of equation (15a) establishes that (to leading order) $\mathcal{M}$ decays exponentially. Using an exponential best fit determines the only free parameter in the system, $\delta=0.264$. This value was used in the numerical simulations of the dissipative NLS equation and the viscous Dysthe system.

Table 2 contains the following quantitative measure of the differences (in centimeters) between the experimental data and PDE predictions

$$
\operatorname{diff}_{n}^{\mathrm{PDE}}=\sum_{j=1}^{J}|2| a_{n}^{\operatorname{expt}}(50 j)|-2| a_{n}^{\mathrm{PDE}}(50 j)||,
$$

where $a_{n}^{\operatorname{expt}}(x)$ and $a_{n}^{\mathrm{PDE}}(x)$ represent the experimentally measured and numerically predicted (using the model PDE) amplitudes of the $n^{\text {th }}$ wave at the gauge located $x \mathrm{~cm}$ from the first wave gauge, $J=11$ for the moderate-amplitude experiment, and $J=10$ for the large-amplitude experiment. 


\begin{tabular}{|c|c|c|}
\hline parameter & symbol & value \\
\hline frequency of carrier wave (rad/sec) & $\omega_{0}$ & 20.94 \\
wave number of carrier wave (cm $\left.{ }^{-1}\right)$ & $k_{0}$ & 0.447 \\
frequency separating sidebands (rad/sec) & $\Delta \omega$ & 1.07 \\
initial amplitude of carrier wave (cm) & $a_{0}$ & $(5.78+9.15 i) * 10^{-2}$ \\
initial amplitude of first upper sideband (cm) & $a_{1}$ & $(0.93-1.33 i) * 10^{-2}$ \\
initial amplitude of second upper sideband (cm) & $a_{2}$ & $(-0.28-0.11 i) * 10^{-2}$ \\
initial amplitude of third upper sideband (cm) & $a_{3}$ & $(-0.03+0.04 i) * 10^{-2}$ \\
initial amplitude of first lower sideband (cm) & $a_{-1}$ & $(0.35-1.38 i) * 10^{-2}$ \\
initial amplitude of second lower sideband (cm) & $a_{-2}$ & $(0.03+0.24 i) * 10^{-2}$ \\
initial amplitude of third lower sideband $(\mathrm{cm})$ & $a_{-3}$ & $(0.04+0.02 i) * 10^{-2}$ \\
wave steepness (dimensionless) & $\epsilon=2\left|a_{0}\right| k_{0}$ & $9.68 * 10^{-2}$ \\
viscosity coefficient (dimensionless) & $\delta$ & 0.264 \\
\hline
\end{tabular}

Table 1: Experimental parameters for the moderate-amplitude experiment.
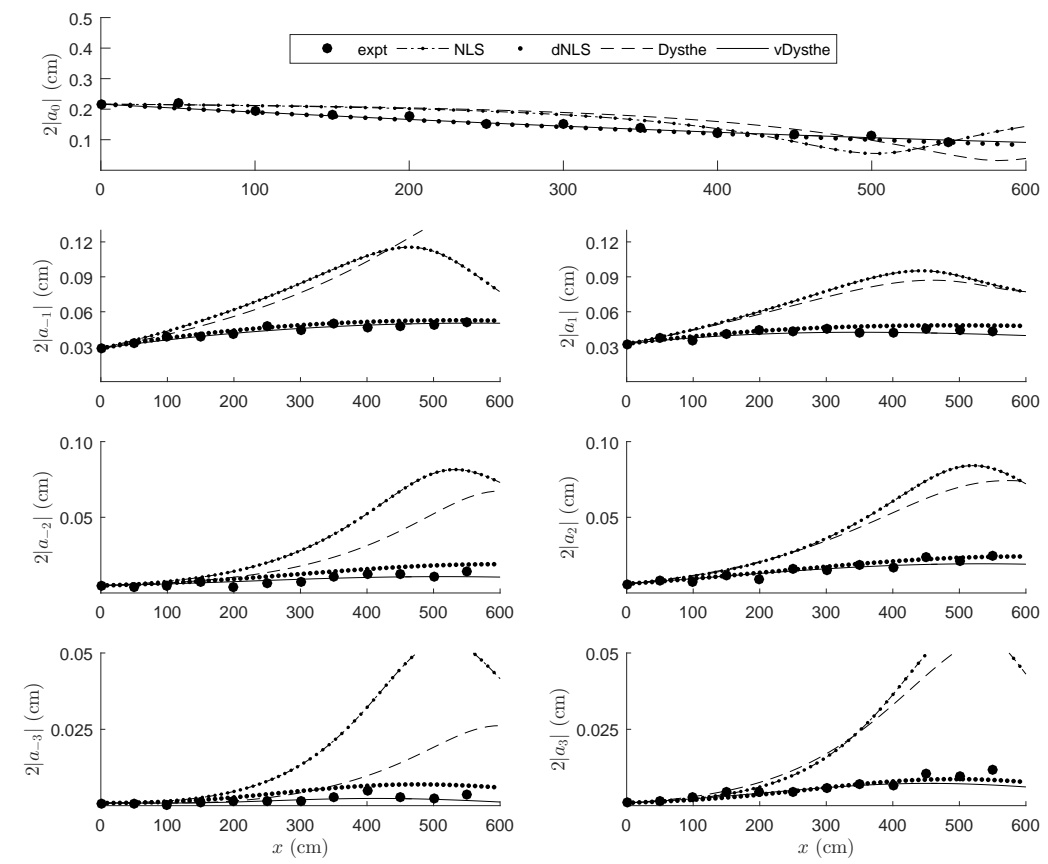

Figure 1: Plots of the amplitudes (in $\mathrm{cm}$ ) of the carrier wave and the six nearest sidebands versus the distance (in $\mathrm{cm}$ ) from the first wave gauge for the moderate-amplitude experiment. 

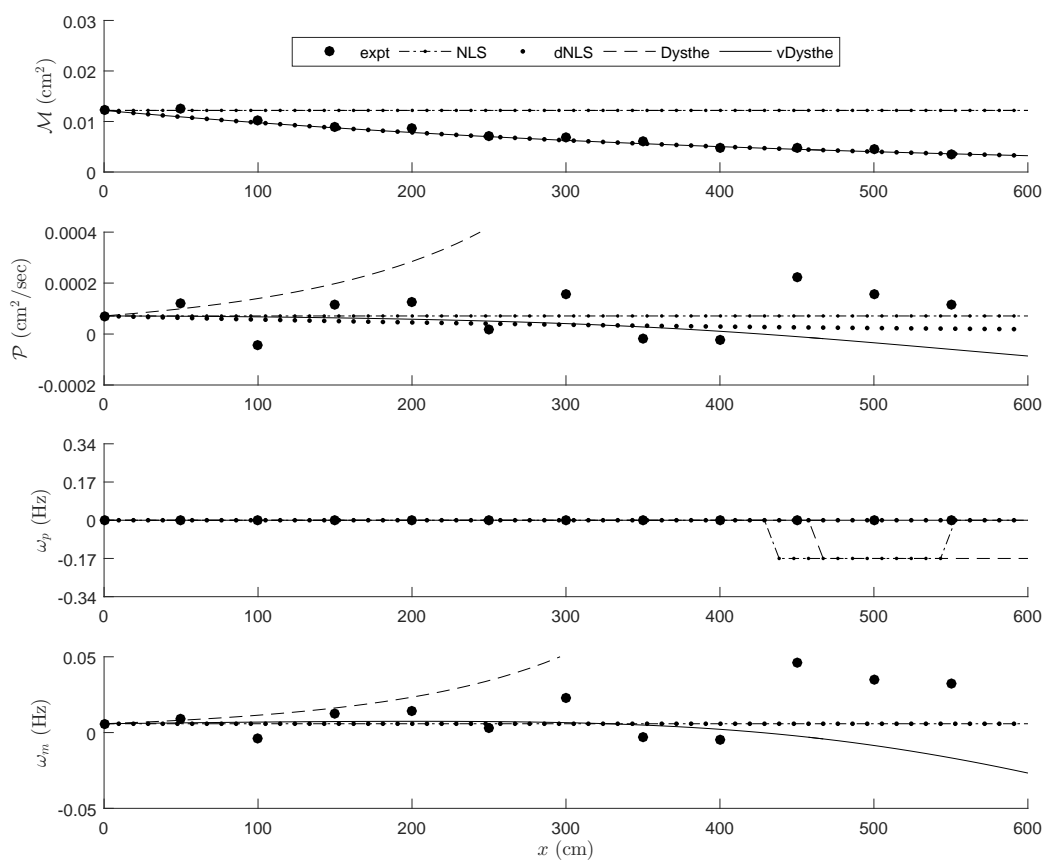

Figure 2: Plots of $\mathcal{M}, \mathcal{P}, \omega_{p}$, and $\omega_{m}$ versus the distance (in $\mathrm{cm}$ ) from the first wave gauge for the moderate-amplitude experiment.

\begin{tabular}{|c|ccccccc|}
\hline PDE & $n=0$ & $n=-1$ & $n=1$ & $n=-2$ & $n=2$ & $n=-3$ & $n=3$ \\
\hline NLS & 0.536 & 0.782 & 0.652 & 0.621 & 0.585 & 0.393 & 0.423 \\
Dysthe & 0.158 & 0.055 & 0.065 & 0.085 & 0.040 & 0.051 & 0.022 \\
dNLS & 0.617 & 0.853 & 0.565 & 0.358 & 0.494 & 0.124 & 0.369 \\
vB & 0.136 & 0.036 & 0.050 & 0.037 & 0.050 & 0.015 & 0.028 \\
\hline
\end{tabular}

Table 2: Comparisons between the experimental data and numerical predictions for the moderate-amplitude experiment using the $\operatorname{diff}_{n}^{\mathrm{PDE}}$ norm that is defined in equation (30). 
Figure 1 and Table 2 establish that the dissipative theories (viscous Dysthe and dissipative NLS) do a much better job modeling the evolution of the amplitudes of the carrier wave and the six nearest sidebands than do the conservative theories (NLS and Dysthe). Both conservative models greatly overpredict the growth of the sidebands. The $\mathrm{vB}$ system predictions are more accurate than the dissipative NLS predictions for five of the seven amplitudes. The differences between the $\mathrm{vB}$ system and dissipative NLS predictions in the other two cases are within experimental error. The vA system (results not shown) provides predictions that are qualitatively similar to the $\mathrm{vB}$ predictions.

Figure 2 shows that the dissipative theories do a much better job predicting the evolution of $\mathcal{M}$ than do the conservative theories. The NLS equation incorrectly predicts a temporary downshift in the spectral peak at $x \approx 425$. The Dysthe system incorrectly predicts a downshift in the spectral peak that starts at $x \approx 450$ and lasts beyond the length on the tank. The Dysthe system predicts increases in $\mathcal{P}$ and $\omega_{m}$ that were not observed in the experiment. It is not possible to determine if the NLS equation, dissipative NLS equation, or viscous Dysthe system provides the best prediction for the spectral mean because all changes are within the bounds of experimental measurement error. However, note that the viscous Dysthe system predicts a small downshift in the spectral mean in the length of the tank. Numerical simulations of the vB system over long tank lengths show permanent FD in both the spectral mean and spectral peak senses. We hypothesize that FD may have been observed in the moderate-amplitude experiment had the wave tank been longer.

\subsection{Large-amplitude experiment}

Table 3 contains the values of the physical parameters for the large-amplitude experiment. Figure 3 contains plots of the Fourier amplitudes versus distance from the first gauge. Table 4 contains the $\operatorname{diff}_{n}^{\mathrm{PDE}}$ comparisons and Figure 4 contains plots of $\mathcal{M}, \mathcal{P}, \omega_{p}$, and $\omega_{m}$ versus $x$. In this experiment, the amplitude of the first lower sideband overtakes that of the carrier wave for the first time somewhere between the gauges at $x=300 \mathrm{~cm}$ and $x=350 \mathrm{~cm}$. The lower sideband overtakes the carrier wave again between the gauges at $x=400 \mathrm{~cm}$ and $x=450 \mathrm{~cm}$. After that, the first lower sideband remains dominant until the end of the experiment. Therefore, FD in the spectral peak sense occurred in this experiment. The spectral mean has a clear downward trend, so FD in the spectral mean sense also occurred. An exponential fit of the experimental $\mathcal{M}$ data determines $\delta=0.321$.

Figure 3 and Table 4 show that the vB system provides the most accurate prediction for the evolution for six of the seven amplitudes. The dissipative NLS equation does a better job for the $n=-1$ mode, though the difference between its predictions and those of the $\mathrm{vB}$ system is within experimental error. Just as in the moderate-amplitude case, the two conservative theories overpredict or greatly overpredict the initial growth of all sidebands.

Figure 4 shows the dissipative theories more accurately model the evolution of $\mathcal{M}$ than do the conservative theories. The Dysthe system predicts a dramatic increase in $\mathcal{P}$ while the vB system predicts a brief upshift followed by a 


\begin{tabular}{|c|c|c|}
\hline parameter & symbol & value \\
\hline frequency of carrier wave $(\mathrm{rad} / \mathrm{sec})$ & $\omega_{0}$ & 20.94 \\
wave number of carrier wave $\left(\mathrm{cm}^{-1}\right)$ & $k_{0}$ & 0.447 \\
frequency separating sidebands (rad/sec) & $\Delta \omega$ & 1.07 \\
initial amplitude of carrier wave $(\mathrm{cm})$ & $a_{0}$ & $(-5.71+8.81 i) * 10^{-2}$ \\
initial amplitude of first upper sideband $(\mathrm{cm})$ & $a_{1}$ & $(0.09-3.41 i) * 10^{-2}$ \\
initial amplitude of second upper sideband (cm) & $a_{2}$ & $(0.69+1.44 i) * 10^{-2}$ \\
initial amplitude of third upper sideband (cm) & $a_{3}$ & $(-0.36-0.27 i) * 10^{-2}$ \\
initial amplitude of first lower sideband (cm) & $a_{-1}$ & $(1.02+3.34 i) * 10^{-2}$ \\
initial amplitude of second lower sideband (cm) & $a_{-2}$ & $(0.89+0.16 i) * 10^{-2}$ \\
initial amplitude of third lower sideband $(\mathrm{cm})$ & $a_{-3}$ & $(-0.15-0.13 i) * 10^{-2}$ \\
wave steepness (dimensionless) & $\epsilon=2\left|a_{0}\right| k_{0}$ & $9.40 * 10^{-2}$ \\
viscosity coefficient (dimensionless) & $\delta$ & 0.321 \\
\hline
\end{tabular}

Table 3: Experimental parameters for the large-amplitude experiment.

\begin{tabular}{|c|ccccccc|}
\hline PDE & $n=0$ & $n=-1$ & $n=1$ & $n=-2$ & $n=2$ & $n=-3$ & $n=3$ \\
\hline NLS & 0.5830 & 0.2929 & 0.2983 & 0.3092 & 0.2149 & 0.2452 & 0.3948 \\
Dysthe & 0.4801 & 0.0921 & 0.0951 & 0.2437 & 0.1752 & 0.1618 & 0.1064 \\
dNLS & 0.5525 & 0.4056 & 0.2973 & 0.3799 & 0.2247 & 0.1591 & 0.2274 \\
vB & 0.3964 & 0.0940 & 0.0406 & 0.1455 & 0.0818 & 0.0127 & 0.0827 \\
\hline
\end{tabular}

Table 4: Quantitative comparisons between the experimental data and numerical predictions for the large-amplitude experiment using the $\operatorname{diff}_{n}^{\mathrm{PDE}}$ norm defined in equation (30).

downward trend. The vB system predicts that the amplitude of the first lower sideband will overtake that of the carrier wave at $x \approx 225 \mathrm{~cm}$. This is earlier than what was observed in the experiment and is related to the fact that the $\mathrm{vB}$ system overpredicts the amplitudes of the first and second lower sidebands. The NLS equation and Dysthe system both predict a temporary downshift in the spectral peak. As expected, NLS and dissipative NLS equations predict that the spectral mean is constant. The vB system predicts a temporary small upshift in the spectral mean followed by a downward trend that follows the experimental data. The downshift predicted by the $\mathrm{vB}$ system is permanent over the length scales examined. In summary, the $\mathrm{vB}$ system provides the most accurate model for all four quantities in this figure.

The accuracy of the vB system comes from a combination of the higher-order viscous term and the higher-order nonlinear terms. Predictions from the NLS equation with the two viscous terms added (results not shown) are much less accurate than those from the vB system.

We are grateful to Maura Brunetti, Shusen Ding, Diane Henderson, and Harvey Segur for helpful discussions. We are also grateful to the referees for their valuable comments and suggestions. This material is based upon work supported by the National Science Foundation under grant DMS-1107476. 

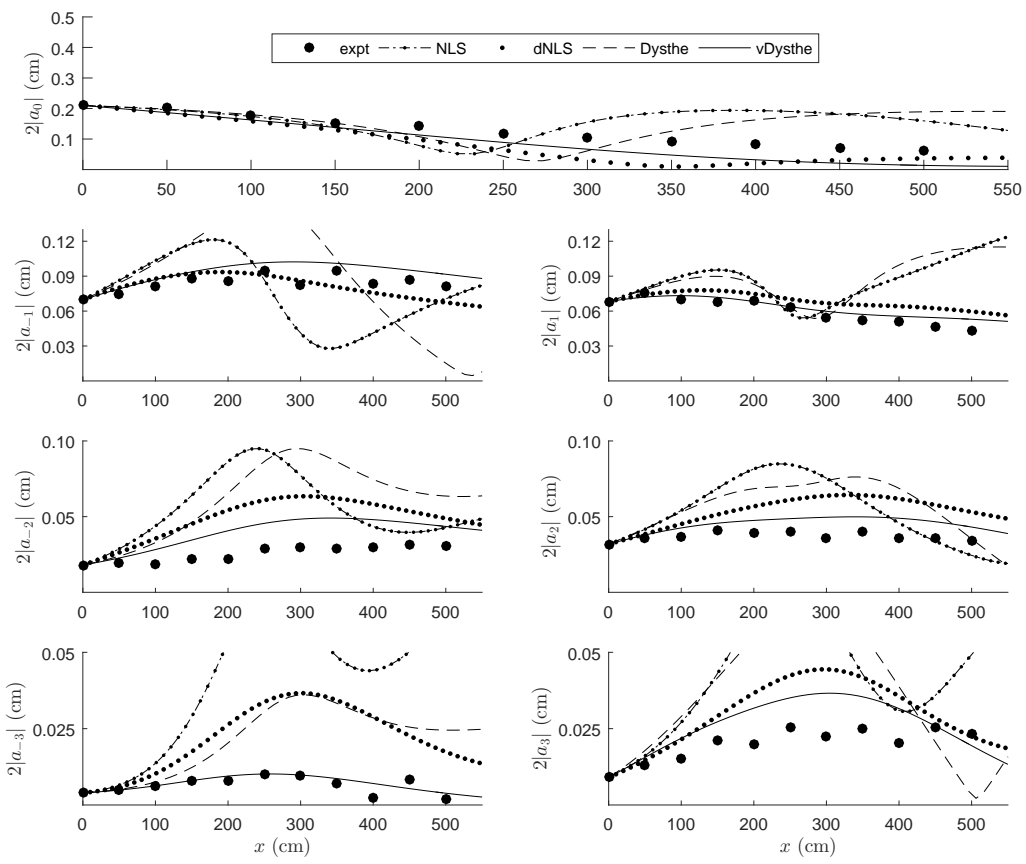

Figure 3: Plots of the amplitudes (in $\mathrm{cm}$ ) of the carrier wave and the six nearest sidebands versus the distance (in $\mathrm{cm}$ ) from the first wave gauge for the large-amplitude experiment. 

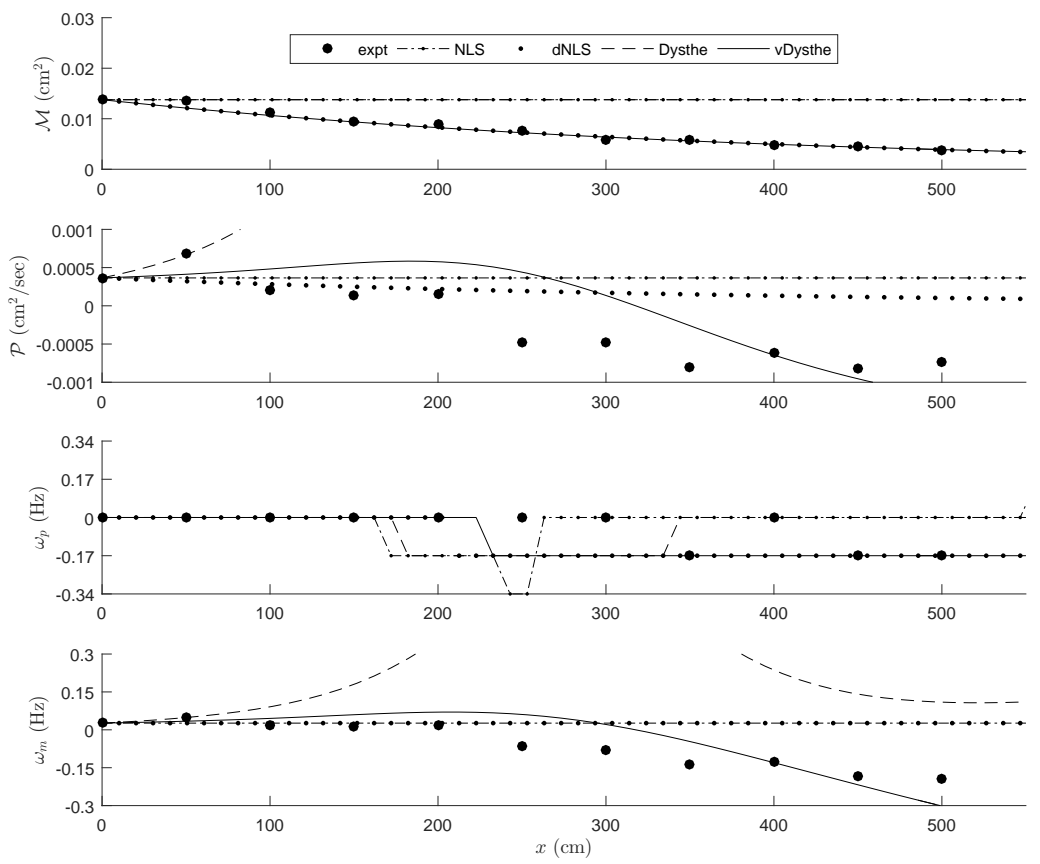

Figure 4: Plots of $\mathcal{M}, \mathcal{P}, \omega_{p}$, and $\omega_{m}$ versus the distance (in $\mathrm{cm}$ ) from the first wave gauge for the large-amplitude experiment. 
[1] K. B. Dysthe, Note on a modification to the nonlinear Schrödinger equation for application to deep water waves, Proceedings of the Royal Society of London A 369 (1979) 105-114.

[2] F. Dias, A. I. Dyachenko, V. E. Zakharov, Theory of weakly damped freesurface flows: A new formulation based on potential flow solutions, Physics Letters A 372 (2008) 1297-1302.

[3] B. M. Lake, H. C. Yuen, H. Rungaldier, W. E. Ferguson, Nonlinear deep water waves: theory and experiment. Part 2. Evolution of a continuous wave train, Journal of Fluid Mechanics 83 (1977) 49-74.

[4] B. M. Lake, H. C. Yuen, A note on some nonlinear water-wave experiments and the comparison of data with theory, Journal of Fluid Mechanics 83 (1977) $75-81$.

[5] M. Y. Su, M. Bergin, P. Marler, R. Myrick, Experiments on nonlinear instabilities and evolution of steep gravity-wave trains, Journal of Fluid Mechanics 124 (1982) 45-72.

[6] W. K. Melville, The instability and breaking of deep-water waves, Journal of Fluid Mechanics 115 (1982) 165-185.

[7] H. Segur, D. Henderson, J. D. Carter, J. Hammack, C. Li, D. Pheiff, K. Socha, Stabilizing the Benjamin-Feir instability, Journal of Fluid Mechanics 539 (2005) 229-271.

[8] V. E. Zakharov, Stability of periodic waves of finite amplitude on the surface of a deep fluid, Journal of Applied Mechanics and Technical Physics 9 (2) (1968) 190-194.

[9] E. Lo, C. C. Mei, A numerical study of water-wave modulation based on a higher-order nonlinear Schrödinger equation, Journal of Fluid Mechanics 150 (1985) 395-416.

[10] K. Trulsen, K. B. Dysthe, Frequency down-shift through self modulation and breaking, in: NATO ASI Series 178, 1990, pp. 561-572.

[11] T. Hara, C. C. Mei, Frequency downshift in narrow banded surface waves under the influence of wind, Journal of Fluid Mechanics 230 (1991) 429477.

[12] Y. Kato, M. Oikawa, Wave number downshift in modulated wavetrain through a nonlinear damping effect, Journal of the Physical Society of Japan 64 (1995) 4660-4669.

[13] M. Brunetti, J. Kasparian, Modulational instability in wind-forced waves, Physics Letters A 378 (2014) 3626-3630.

[14] M. Brunetti, N. Marchiando, N. Berti, J. Kasparian, Nonlinear fast growth of water waves under wind forcing, Physics Letters A 378 (2014) 1025-1030. 
[15] A. Islas, C. M. Schober, Rogue waves and downshifting in the presence of damping, Natural Hazards and Earth System Sciences 11 (2011) 383-399.

[16] K. Trulsen, K. B. Dysthe, Frequency downshift in three-dimensional wave trains in a deep basin, Journal of Fluid Mechanics 352 (1997) 359-373.

[17] J. D. Carter, Stability and existence of traveling wave solutions of the two-dimensional nonlinear Schrödinger equation and its higher-order generalizations, Ph.D. thesis, University of Colorado at Boulder (2001).

[18] H. Segur, D. M. Henderson, J. L. Hammack, Can the Benjamin-Feir instability spawn a rogue wave?, in: P. Müller, D. M. Henderson (Eds.), Proceedings of the 14th 'Aha Huliko'a Hawaiian Winter Workshop Honolulu, Hawaii, 2005, pp. 43-57.

[19] O. Gramstad, K. Trulsen, Hamiltonian form of the modified nonlinear Schrödinger equation for gravity waves on arbitrary depth, Journal of Fluid Mechanics 670 (2011) 404-426.

[20] E. A. Coddington, N. Levinson, Theory of Ordinary Differential Equations, McGraw-Hill, New York, 1955.

[21] T. J. Bridges, F. Dias, Enhancement of the Benjamin-Feir instability with dissipation, Physics of Fluids 19 (2007) 104104.

[22] J. D. Carter, C. C. Contreras, Stability of plane-wave solutions of a dissipative generalization of the nonlinear Schrödinger equation, Physica D 237 (2008) 2392-2396. 